\title{
How wave physics values diversity and interconnection
}

\author{
Ocean Mercier
}

\begin{abstract}
The target article "The double spiral and ways of knowing" presents a striking example of how wave physics can be understood in relation to Māori ways of knowing. The wiriwiri is innovatively represented by a wave form reminiscent of a physicist's 'wave packet'. In this commentary I use this evocative imagery to highlight some of the more basic aspects of 'wave interference'. In doing so, I draw attention to the importance of individual diversity but social interconnection. These are principles that physics seems to agree with, albeit in its own mathematical and graphical language.
\end{abstract}

Keywords: frequency; waves

"Spiral dynamics and ways of knowing" (Williams \& Henare, 2009) presents an innovative juxtapositions of physics and Maori knowledge. The concepts of spiral dynamics and Māori values seem to resonate quite naturally with each other, demanding further examination and discussion. The representation of the wiriwiri in Figure 1 of that paper is reminiscent of a 'wave packet', and has invited me to create and deconstruct a wave packet, reveal its make up and reflect upon what happens, in general, when waves of diverse frequencies are added together.

I have used Labview-based programmes from the Wave Simulations suite (Information about acquiring this free resource is available from The New Zealand Physics Teachers' Resource Bank at http://www.physics.school.nz) to produce a companion figure to figure 1 of the target article. Figure 1 of this article shows the amplitude versus time graph of two high-pitched sound frequencies. Through what is known as a Fourier transform, the information in the top panel can be re-presented in a drastically different way, with oscillation 'frequency' on the xaxis. The standard unit for frequency is an inverted second, otherwise known as a Hertz (Hz). The two sharp peaks at the frequencies $4250 \mathrm{~Hz}$ and $4500 \mathrm{~Hz}$ in the second panel of figure 1 are the frequencies interacting to create the wave packet. This commonality of shape suggests that the wave form presented in the target article may have arisen from the interaction of two waves. The bottom two panels show what these waves look like. They are virtually indistinguishable because they are so close in frequency, a characteristic which gives rise to the 'wave packet' shape and the audio phenomenon of 'beats'. Nonetheless, the close observer will note the appearance of more peaks in the higher-frequency wave form. Note also, that these waves assume the regular sinusoidal shape that characterises natural harmonic motion. 
Mai Review, 2009, 3, Peer Commentary 4

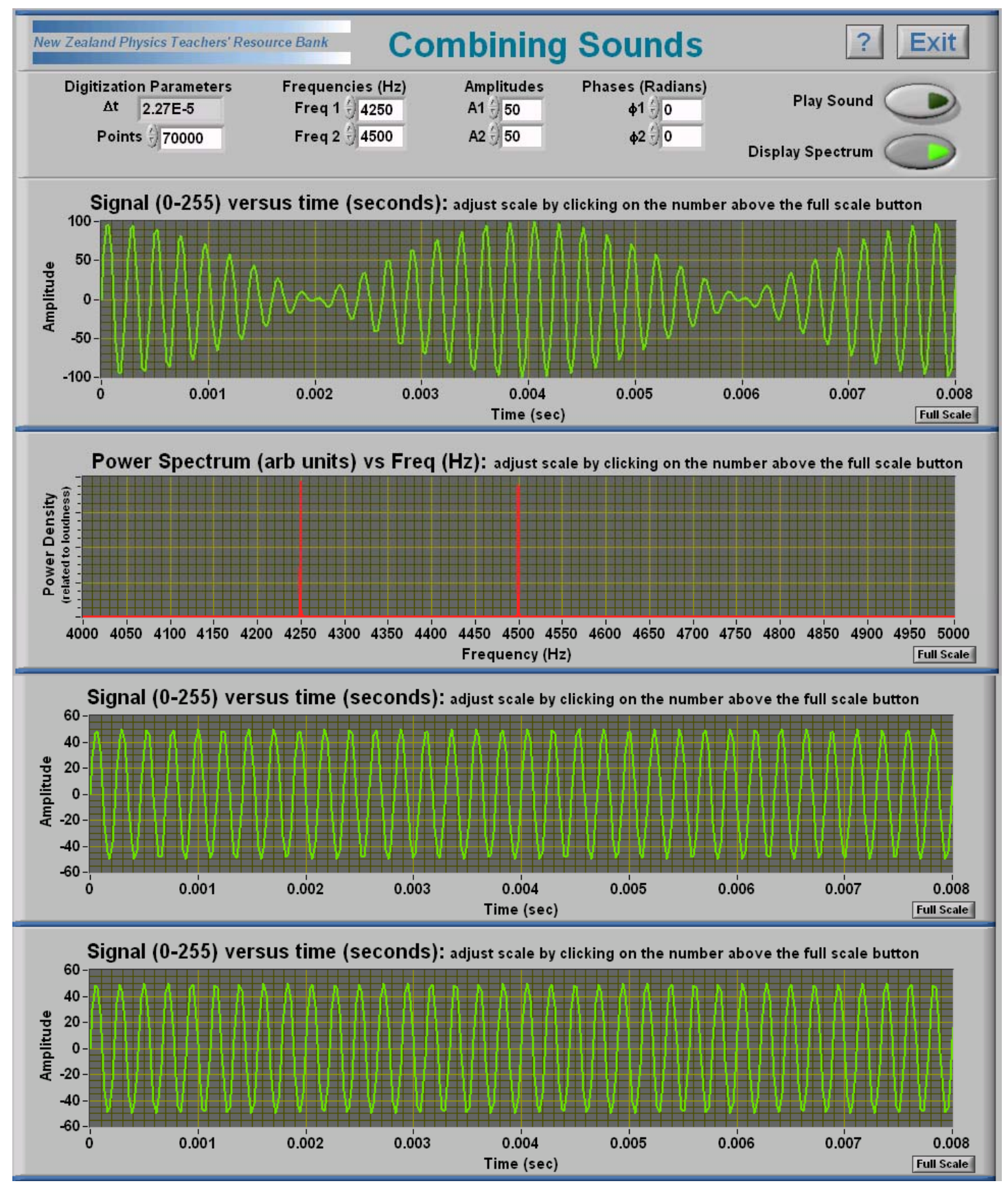

Figure 1. Screen captures from the Combining Sounds programme in the Wave Simulations software. Top panel: a 'wave packet', resulting from the interaction of two similar frequencies $4250 \mathrm{~Hz}$ and $4500 \mathrm{~Hz}$ : and second panel: deconstruction (Fourier transform) of the wave form to show spikes at the two different wave frequencies. The two bottom panels show the sinusoidal waveforms for $4250 \mathrm{~Hz}$ and $4500 \mathrm{~Hz}$ respectively.

What if we add in more frequencies? While this question has little relevance to the representation of a wiriwiri, it will illustrate a phenomenon that is considered fundamental to physics, and can also be considered in light of its resonance with one of our key values, that of diversity. 

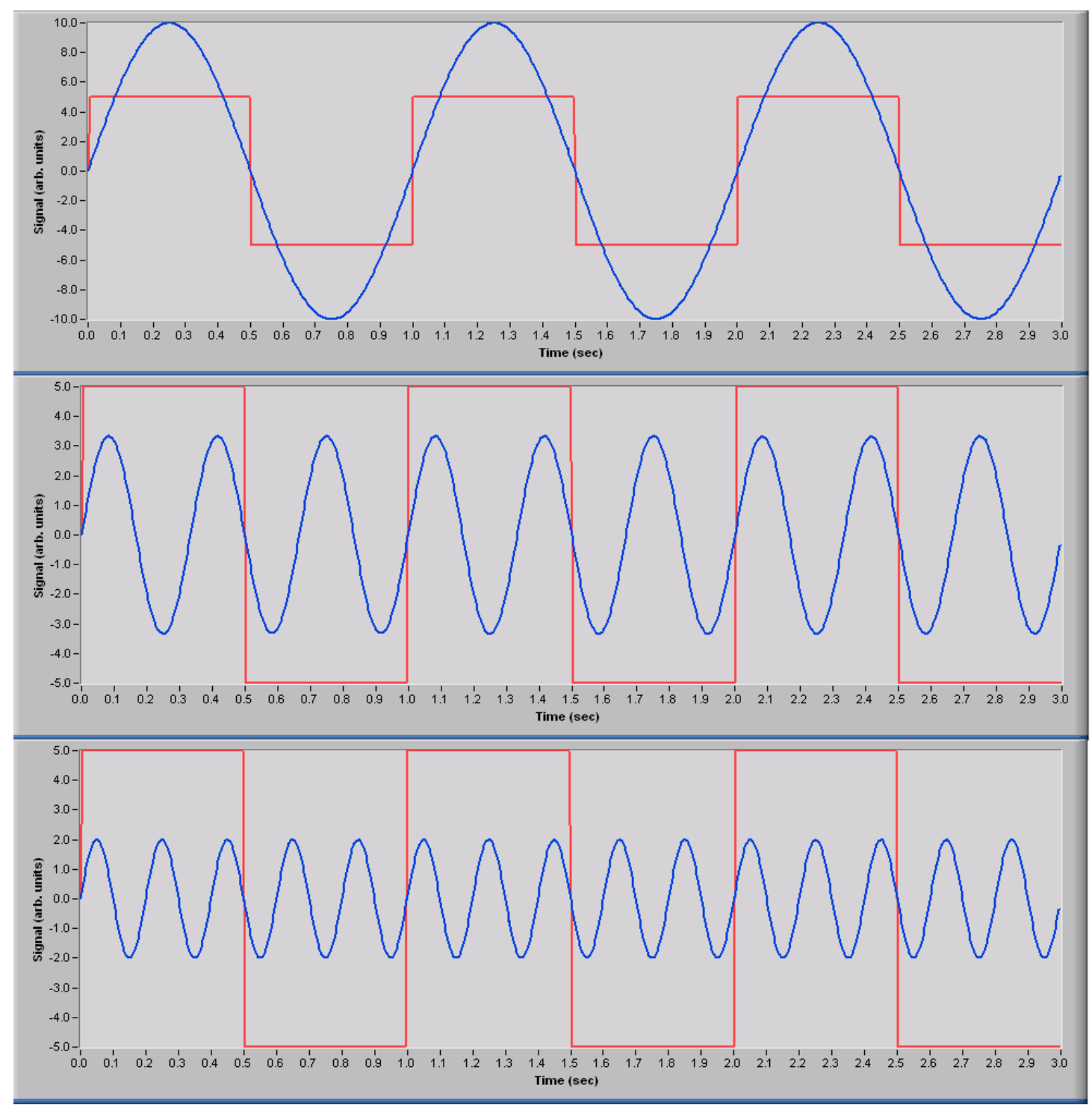

Figure 2. Screen captures from the SquareWave programme of the Wave Simulations software. The three sinusoidal waves (top: $1 \mathrm{~Hz}$; middle: $3 \mathrm{~Hz}$; and bottom: $5 \mathrm{~Hz}$ ) can interact to produce an approximation of the square wave indicated in red (see figure 3).

The three panels of figure 2 show three sinusoidal waves (such as those in the bottom panels of figure 1). The red square wave is provided for scale reference. Note that the $1 \mathrm{~Hz}$ wave in the top panel has a y-scale that runs from -10 to +10 , whereas the other waves are in panels of scale -5 to +5 . The $1 \mathrm{~Hz}$ wave has an amplitude of 10 , the $3 \mathrm{~Hz}$ wave an amplitude of 3.33 and the $5 \mathrm{~Hz}$ wave an amplitude of 2 . The relativity of these amplitudes is described mathematically by the so-called Fourier series.

In the top panel of figure 3 you will see the wave form that has resulted from the interaction of the three sinusoidal waves of different frequency. Looking at the top panel you might observe that the new wave shape looks somewhat square. The bottom panel illustrates how we might make the wave even squarer by the strategic addition of five new waves of varying frequencies and amplitudes. It is possible to reconstruct any shape of wave (triangular, circular) using specific quantities of certain frequencies. As a rule, the higher the frequency of the wave added to the mix, the smaller that wave's amplitude. This power of physics to reconstruct and recreate from basic sinusoidal waves is reliant on diversity; both of frequency and amplitude. 


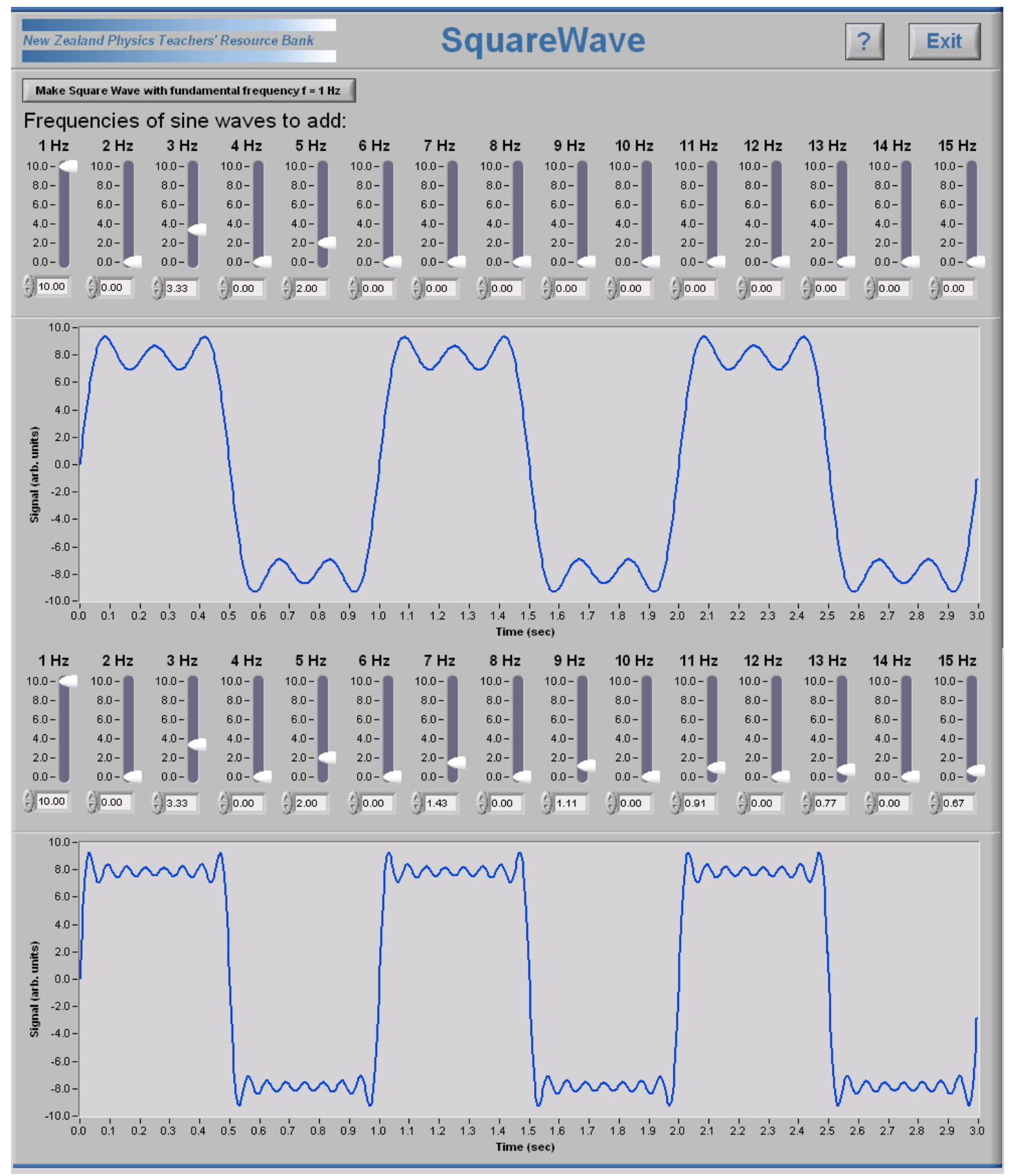

Figure 3. Screen captures from SquareWave.Top panel: an approximate rendition of a square wave form, using three different frequencies (or harmonics) of the $1 \mathrm{~Hz}$ sine wave: and Bottom panel: eight different waves of varying amplitude and frequency gives a closer approximation of a square wave.

Let's now consider what happens when waves of the same frequency (and same amplitude, for convenience) interact. There are two extremes and a continuum of possibilities in between, as illustrated in figure 4. If the waves are 'in phase', meaning their troughs and peaks occur at the same time, the size of the output wave will be the sum of the two individual waves, in this case, doubled. If we were to consider two sound waves interfering in this way, the intensity of the resultant sound would be doubled, as seen in figure 4, part (a). 


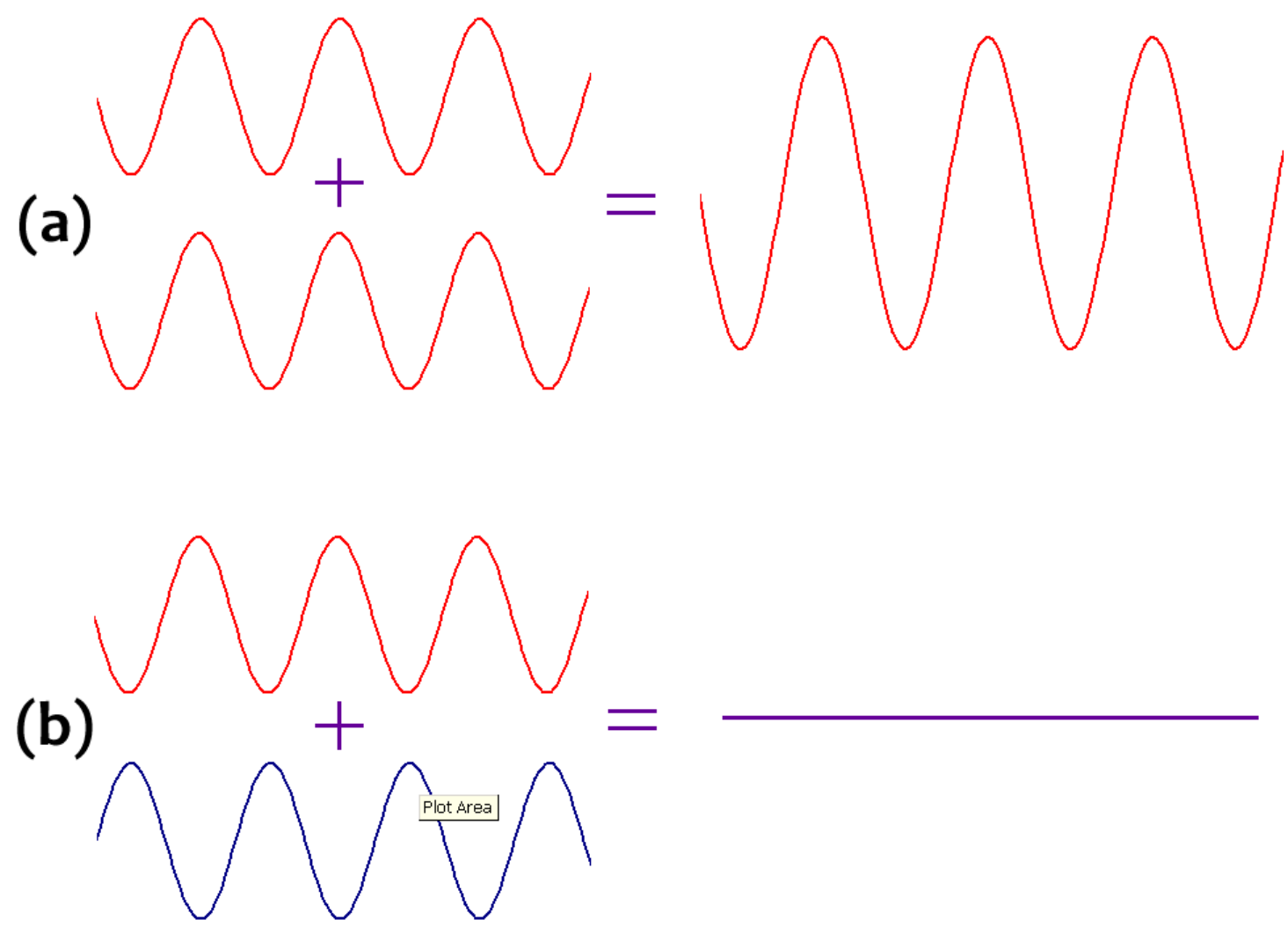

Figure 4. Types of wave interference when two waves of (or on) the same frequency and amplitude interact. (a) Total constructive interference (waves 'in phase') doubles the resultant amplitude; and (b) Total destructive interference (waves are 180 degrees out of phase) results in an output amplitude of zero.

If the waves were out of phase by exactly half a cycle (or 180 degrees), the interaction between them would result in complete destructive interference. In panel (b) you observe the resulting wave form: a flat line. At any given moment in time, the two 'frequencies' are pulling in different directions with the same 'strength' and the result is zero amplitude.

If the waves were to get out of phase by any amount other than 0 degrees and half a cycle, the resultant wave would have the same frequency but its amplitude would be somewhere in between complete constructive and destructive interference.

The lesson we learn from physics is that the collaboration of diverse wave types, entities on 'different wavelengths', is key to the creation and construction of new forms, new representations and, arguably, new knowledge. (I've switched to talking about wavelengths in this section, but it is just the counterpart to frequency.) This idea has an additional analogue in the area of interdisciplinary research, in which collaboration of different disciplines is seen as crucial to new and creative understandings of the world around us. Indeed, it's actually impossible to reconstruct other types of wave using only waves of the same frequency, they have to be on a 'different wavelength' in order to reconstruct something new.

Waves interacting with the same wavelength have the potential for self-destruction, especially if they get out of phase (or out of sync) with each other. By contrast, waves interacting with other waves of different wavelengths will never result in complete destructive interference, no matter how 'out of sync' the waves are. As seen in the 'wiriwiri', there may be lulls (or minima, as physicists call the pinched ends of the wave packet) but these will always be localised in time and will only come after a space and time of noise and productivity. 
Mai Review, 2009, 3, Peer Commentary 4

The need for diversity (of wavelength) and interconnection (the waves interact to produce a new waveform) by physics could be seen as analogous to valuing diversity in society. The inter-relationship of two types of oscillation in the wiriwiri is a striking way to see that by working together from our own unique and individual spaces, we breed creativity and new knowledge. By being the same, however, we risk stymieing our creative interactive potential.

\section{Reference}

Williams, L.R.T., \& Henare, M. (2009). The double spiral and ways of knowing. MAI Review, 3, Target Article 3, 9 pages

\section{Author Notes}

Dr Ocean Mercier (Ngāti Porou) is a Lecturer in Te Kawa a Māui - School of Māori Studies at the Victoria University of Wellington.

E-mail: ocean.mercier@vuw.ac.nz 